\title{
Coolest Path: Spectrum Mobility Aware Routing Metrics in Cognitive Ad Hoc Networks
}

\author{
Xiaoxia Huang*, Dianjie $\mathrm{Lu}^{*}$, Pan $\mathrm{Li}^{\dagger}$ and Yuguang Fang $\ddagger \S$ \\ *Shenzhen Institutes of Advanced Technology, Chinese Academy of Sciences, Shenzhen, China \\ ${ }^{\dagger}$ Department of Electrical and Computer Engineering, Mississippi State University, MS 39762 \\ ${ }^{\ddagger}$ Department of Electrical and Computer Engineering, University of Florida, FL 32611

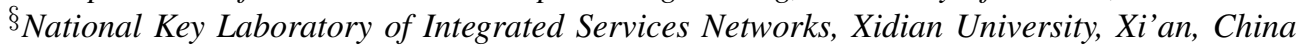 \\ Email: \{xx.huang,dj.lu\}@siat.ac.cn, li@ece.msstate.edu,fang@ece.ufl.edu
}

\begin{abstract}
Cognitive Radio (CR) emerges as a promising solution to current unbalanced spectrum utilization. The cognitive ad hoc network can take advantage of dynamic spectrum access and spectrum diversity over wide spectrum. It could achieve higher network capacity compared to traditional ad hoc networks, thus supporting bandwidth-demanding applications. A cognitive radio operates over wide spectrum with unpredictable channel availability. Moreover, the transmission opportunity of a cognitive node is not guaranteed due to the presence of primary users (PUs). These two unique features define new routing problems in cognitive ad hoc networks. To better characterize the unique features of cognitive radio networks, we propose new routing metrics, including accumulated spectrum temperature, highest spectrum temperature, and mixed spectrum temperature to account for the timevarying spectrum availability. The proposed metrics favor the "coolest" path, or the path with the most balanced and/or the lowest spectrum utilization by the primary users. We also study the computational complexity of the routing algorithm in cognitive ad hoc networks. Experiment results on our USRP2 testbed show that the proposed metrics are capable of capturing the fluctuation of spectrum availability and suitable for cognitive ad hoc networks.
\end{abstract}

Keywords-cognitive radio; spectrum mobility; routing

\section{INTRODUCTION}

Due to the fixed spectrum management, current spectrum utilization is temporally and spatially unbalanced, ranging from $15 \%$ to $85 \%$ [1]. Some portions of the spectrum, such as the $2.4 \mathrm{GHz}$ ISM band becomes noticeably congested due to the widely used WiFi and Bluetooth devices. But frequencies licensed for television broadcast are underutilized in general. The spectrum scarcity problem is caused by the uneven spectrum utilization. Cognitive Radio (CR) [2]- [4] emerges as a promising solution to this problem as it can opportunistically access the white space without interfering with primary users (PUs). Therefore, the cognitive ad hoc networks can take advantage of dynamic spectrum access and spectrum diversity over wide spectrum. It could achieve higher network capacity compared to traditional ad hoc networks, thus supporting bandwidth-demanding applications.

Different from multi-channel multi-radio networks [5] [6], cognitive ad hoc networks operate over wide spectrum with unpredictable channel availability. The channels are known to all nodes in muti-channel multi-radio networks. But a cognitive node has no priori knowledge about the frequency channels to be used due to the spectrum dynamics. After spectrum sensing, channels that could be used for communications are assigned through spectrum management without degrading the link quality of primary users. Another feature is that the transmission opportunity of cognitive nodes is not guaranteed. The transmission of cognitive node will be interrupted if a primary user appears on the borrowed licensed channel. Cognitive nodes must cease transmission and vacate the licensed band for primary users. The two unique features introduce new routing problems in cognitive ad hoc networks.

In cognitive ad hoc networks, a cognitive node transmits over time-varying frequency bands because the channel availability is subject to the activities of primary users. Existing routing metrics [7] [8] which are proposed for wireless networks with fixed operating channel sets do not consider the spectrum dynamics, and hence are not suitable for cognitive radio networks. As spectrum handover caused by the appearance of primary users happens frequently in cognitive ad hoc networks, path breakage happens frequently to the cognitive nodes. Therefore, the performance of routing heavily depends on the spectrum allocation and spectrum availability along the path. Different from the routing problem in other wireless networks, routing is inherently tightly coupled with spectrum selection in cognitive ad hoc networks. The routing problem is actually composed of two subproblems, i.e., choosing the appropriate path and choosing the frequency bands along the path. The two subproblems are dependent on each other. Spectrum availability decides path selection, while the path choice affects the allocation of the available spectrum. Thereupon, routing is an inherently complicated problem in cognitive radio networks.

To improve the routing performance in cognitive ad hoc networks, a novel routing metric is essential to minimize the impact of spectrum takeover by the primary users. The contribution of the paper is threefold. First, to better 
characterize the unique features of cognitive radio networks, we propose new routing metrics, including accumulated spectrum temperature, highest spectrum temperature, and mixed spectrum temperature, to account for the time-varying spectrum availability. Mixed spectrum temperature is a twodimensional routing metric, which is a tuple of accumulated spectrum temperature and highest spectrum temperature. The proposed routing metrics favor the path with the most balanced and/or lowest spectrum utilization by the primary users. The optimal path is of good path stability and is relatively immune to the variation of spectrum utilization by the primary users. Second, we study the computational complexity of routing in cognitive radio networks. Third, we investigate the performance of the proposed routing metrics on our USRP-2 testbed.

The paper is organized as follows. Section II defines the routing metrics which capture the variation of the spectrum availability caused by the primary users. Section III presents routing algorithms which can find the optimal path efficiently employing the proposed routing metrics. In Section IV we present our implementation of the new routing algorithms on the USRP-2 testbed, and show the experiment results. Section V discusses the previous work on related topics. Finally, Section VI concludes the paper.

\section{Spectrum Mobility Aware Routing Metrics}

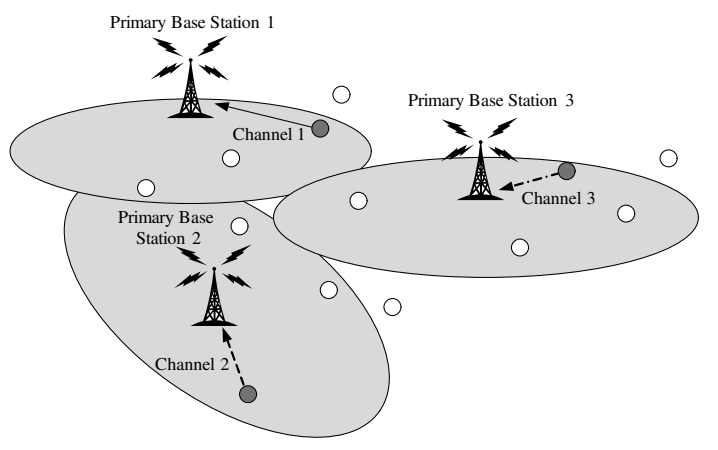

Figure 1. Coexistence of multiple legacy systems

We consider that multiple wireless legacy systems coexist in the same area as shown in Fig. 1. Different types of primary users (PUs) and secondary users (SUs) coexist. Let the shaded dot denote legacy wireless devices, and the empty dot denote the device with a cognitive radio. The primary users have the access right of the corresponding licensed band. A secondary user can only opportunistically access the licensed bands if it does not interfere with active primary users. If a primary user is detected on the licensed band, the secondary user should evacuate immediately. It should switch to another frequency band to resume the transmission or simply defer the unfinished transmission until the licensed band becomes free. The cognitive nodes listen on the licensed bands to monitor the spectrum usage of primary users. They contend on a free frequency band before initiating a transmission. The cognitive nodes can transmit over multiple non-contiguous frequency bands simultaneously to meet the bandwidth requirement. We use "node" and "user" interchangeably in this paper.

\section{A. Spectrum Mobility}

Different from that in multi-channel wireless networks, the spectrum availability in cognitive radio networks varies in time and space. Cognitive nodes must not affect the performance of primary users. In contrast, all nodes in multichannel wireless networks share all the channels. Hence, routing in cognitive ad hoc networks is subject to the fluctuation of spectrum availability due to the appearance of primary users. When a relaying secondary user is transmitting over the licensed band, the transmission will be interrupted if a primary user appears on that band. In this situation, the secondary user has to perform spectrum mobility if a qualifying channel is available. Spectrum mobility is defined as the migration of the cognitive node over the spectrum. In a mobile cognitive ad hoc network, spectrum mobility could be triggered by node mobility. Since the spectrum usage varies in space, the movement of primary nodes affects the temporal spectrum availability. Spectrum mobility is also invoked if a cognitive node moves into the transmission range of a primary user. Additionally, energy consumption is of great concern in cognitive ad hoc networks. Cognitive radio has to monitor the activities over a wide spectrum which incurs high energy cost. Therefore, energy efficiency is a significant factor in cognitive ad hoc networks. Since frequent rerouting and spectrum mobility are adverse to energy efficiency, a routing algorithm which is insensitive to the fluctuation of spectrum availability is desired.

To smoothly perform spectrum mobility, the interrupted cognitive node should promptly find an available channel to continue the transmission to its next-hop node. If there is no free common channel between them, then path repair or rerouting happens. The interrupted cognitive node has to find another path to the destination. Frequent spectrum mobility causes unstableness in routing, which results in long delay and heavy packet loss, and hence should be thoroughly considered when designing routing protocols in cognitive ad hoc networks. Spectrum mobility aware routing in cognitive ad hoc network significantly differs from routing in ad hoc networks. The network topology in cognitive ad hoc networks is decided by the location of nodes and the time-varying spectrum availability at each node. In contrast, the network topology is basically decided by the geographic location of nodes in ad hoc networks. Since a routing algorithm heavily relies on the underlying network topology, the efficiency of a routing algorithm in cognitive ad hoc networks depends on both the distribution of spectrum usage at each node and the geographic distribution of nodes. Therefore, the routing problem in cognitive ad hoc networks 
is much more complicated than its counterpart in ad hoc networks.

\section{B. Path Stability}

We address the problem of routing instability through selecting the path which is most unlikely to experience spectrum mobility. In cognitive ad hoc networks, we could hardly predict the variation of the spectrum usage of all nodes accurately. It is more reasonable to choose the statistically stablest path. The routing problem in cognitive ad hoc networks is different from existing wireless networks because spectrum mobility has significant impact on path quality. Since existing routing metrics are not suitable for cognitive ad hoc networks, we propose a new concept path stability, which accounts for spectrum mobility. Path stability is determined by two factors, frequency diversity and channel stability. Frequency diversity is related to the size of the common channel pool, or sub-link set at each hop over the path. Each common channel between two nodes is called a sub-link. Frequency diversity refers to the minimum number of sub-links over all the hops on the path, which is thus a bottleneck metric. A path with more free sublinks at each hop has higher frequency diversity, and hence is more resilient to spectrum dynamics. As shown in Fig. 2 , when primary user 1 appears on channel 1 , channel 1 is no longer available at node $B$. As a result, the link $(B, C)$ over the shortest path fails. In contrast, the path with more sub-links still works as node $B$ and $D$ could switch to common channel 2 and continue transmission. This link repair procedure is transparent to other links. Channel stability is defined as the probability that the channel is claimed by a primary user. A path with better channel stability experiences less frequent spectrum mobility and path breakage. Taking both frequency diversity and channel stability into consideration, link stability and path stability are defined in Definition 1 and Definition 2 respectively.

Definition 1: Link stability is the probability that the end nodes could communicate with each other.

Definition 2: Path stability is the probability that the path will not experience path breakage due to the variation of available spectrum.

\section{Link Temperature}

First, we introduce some definitions related to link temperature in our paper. The primary channel usage is defined as the channel busyness ratio attributed to primary users. Link temperature is defined as the primary channel utilization at each node. It is used to measure link stability. Apparently, the lower the link temperature is, the better the load balancing is and the less the transmission interruption due to the spectrum handover is.

The link temperature is critical to link stability. It can be calculated as the statistical primary channel busyness ratio. Each cognitive node updates the primary channel busyness

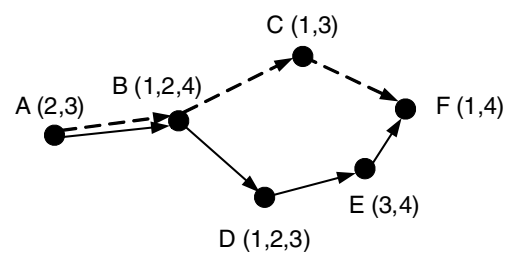

(a) Shortest path (dotted line) and path with higher frequency diversity

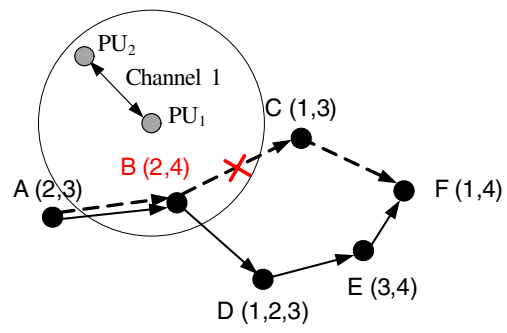

(b) Shortest path fails when the channel 1 becomes unavailable at node $\mathrm{B}$

Figure 2. Impact of frequency diversity on path stability

ratio over time window $W$ for each licensed channel. To accurately calculate the primary channel busyness ratio, cognitive nodes should have the knowledge of the ongoing transmissions of nearby primary nodes. There are two ways to obtain the primary usage statistics of neighbors. One way is to let each primary node maintain the channel usage history information. It calculates its own channel utilization statistics and sends it to neighboring cognitive nodes. Another way is to monitor the transmissions of neighboring primary nodes and compute the primary channel busyness ratio at cognitive nodes. The first method imposes additional requirement on primary users, which is not practical. Although the second method requires the cognitive node to continuously overhear the transmission over the licensed bands, it does not require communications between primary users and cognitive nodes. Therefore, we use the second method to obtain the primary channel busyness ratio in our paper.

Denote by $u_{i}^{c}$ the busyness ratio of channel $c$ caused by primary nodes detected at cognitive node $i$. Cognitive nodes update the channel usage information of its neighboring primary nodes through spectrum sensing. Having separate interference areas, two neighboring nodes may have different observations on the same channel. For example, the primary utilization of channel $c$ at cognitive node $i$ is $u_{i}^{c}$, while cognitive node $j$ may find out the primary utilization of channel $c$ to be $u_{j}^{c}$. Only when the common channel is free at both nodes, they could use the channel for transmission. Denote by $T_{i j}^{c}$ the temperature of channel $c$ between node $i$ and node $j$, it is

$$
T_{i j}^{c}=1-\left(1-u_{i}^{c}\right)\left(1-u_{j}^{c}\right) .
$$

$T_{i j}^{c}$ is the probability that common channel $c$ is not available between $i$ and $j$. Furthermore, the optimal channel between 
$i$ and $j$ is

$$
C_{i j}^{o p t}=\arg \min _{c} T_{i j}^{c} .
$$

Accordingly, the link temperature between two cognitive nodes is defined as follows.

Definition 3: The link temperature $T_{i j}$ is defined as the temperature of the channel with the lowest primary channel temperature between $i$ and $j$

$$
T_{i j}=\min _{c}\left\{T_{i j}^{c}\right\} .
$$

Through periodical exchange of the primary channel busyness ratio with neighboring nodes, each cognitive node could obtain the temperature of all the bands using (1). Among all the bands, the channel with the lowest link temperature is selected for transmission over the hop. The usage of the link temperature in finding the optimal routing is to be described later.

The link temperature metric does not consider the contention among cognitive nodes [9]. If multiple nodes detect a "cool" channel, they may attempt to grab it simultaneously. This problem can be addressed via MAC layer design. The contention between cognitive nodes is the same as that in traditional ad hoc networks. It has been studied extensively in the literature and is beyond the scope of this paper.

\section{Path Temperature}

To quantify the path stability, a novel routing metric, path temperature is introduced. Path temperature refers to the end-to-end spectrum utilization, or spectrum temperature of the selected bands over the path. Some licensed bands are used more heavily by primary users than others, so cognitive nodes using them experience more severe contention and frequent spectrum handover. The path with the lowest path temperature is the "coolest" path, or the stablest path.

The path temperature could be defined in different ways. It could be measured by accumulated spectrum temperature, or/and highest spectrum temperature. We introduce three different path temperature definitions.

1) Accumulated spectrum temperature: The accumulated spectrum temperature is the sum of the link temperatures over the entire path. The accumulated spectrum temperature $T_{a}$ is

$$
T_{a}^{\mathcal{L}}=\sum_{(i, j) \in \mathcal{L}} T_{i j}
$$

where $\mathcal{L}$ is the set of links along the path and is used to denote the path as well. The accumulated spectrum temperature is used to select a path with the lowest endto-end spectrum utilization. To maximize the path stability is $\max \prod_{(i, j) \in \mathcal{L}}\left(1-T_{i j}^{c}\right)$. Note that $\max \prod_{(i, j) \in \mathcal{L}}\left(1-T_{i j}^{c}\right)$ is equivalent to $\max \sum_{(i, j) \in \mathcal{L}} \log \left(1-T_{i j}^{c}\right)$, and hence $\min \left\{-\sum_{(i, j) \in \mathcal{L}} \log \left(1-T_{i j}^{c}\right)\right\}$, which can be approximated by $\min \sum_{(i, j) \in \mathcal{L}} T_{i j}$. For simplicity, we use $\sum_{(i, j) \in \mathcal{L}} T_{i j}$ as the accumulated spectrum temperature in (4).

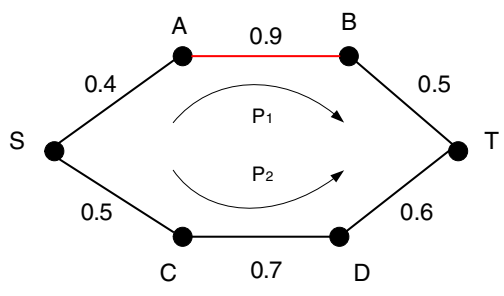

Figure 3. Comparison of paths with different highest spectrum temperature

2) Highest spectrum temperature: The highest spectrum temperature is the maximum primary channel utilization, or maximum link temperature of the links over the path. Highest spectrum temperature $T_{\max }$ is calculated as

$$
T_{\text {max }}^{\mathcal{L}}=\max _{(i, j) \in \mathcal{L}}\left\{T_{i j}\right\} .
$$

The highest spectrum temperature is a bottleneck routing metric. A path with lower highest spectrum temperature may have better link stability at each hop.

3) Mixed spectrum temperature: The accumulated spectrum temperature is used to measure the utilization of all bands used over the path. But solely using the accumulated spectrum temperature is not enough to precisely quantify the path quality. As shown in Fig. 3, path $P_{1}$ has accumulated spectrum temperature of 1.8 , which is the equivalent to path $P_{2}$. However, the path stability of the two paths is not identical. $P_{1}$ has highest spectrum temperature 0.9 , which is higher than that of $P_{2} . P_{1}$ is more prone to breakage at link $(A, B)$ due to the higher probability of spectrum inaccessibility, while $P_{2}$ is more likely to fail at link $(C, D)$. In other words, link $(A, B)$ and link $(C, D)$ are the bottleneck links of $P_{1}$ and $P_{2}$, respectively. Compared to $P_{2}, P_{1}$ is less stable because the link temperature of link $(A, B)$ is higher. Any link failure or link change would impair link stability or even cause path breakage. So a path with lower highest spectrum temperature is less likely to experience link breakage along the path. Therefore, we use the highest spectrum temperature to eliminate the path with extremely unbalanced spectrum utilization and choose the path with better path stability. The two-dimensional path temperature metric favors the path with even link temperature and low total spectrum temperature.

The path with low accumulated spectrum temperature and low highest spectrum temperature has high stability. If there exists no global optimal path, either the path with the lowest accumulated spectrum temperature or the one with the lowest highest spectrum temperature is assumed to be the optimal path $P^{o p t}$.

$P^{o p t}= \begin{cases}\arg \min _{\mathcal{L}}\left\{T_{\text {max }}^{\mathcal{L}}, T_{a}^{\mathcal{L}}\right\}, & \text { if } \mathcal{L} \text { exists } \\ \arg \min _{\mathcal{L}}\left\{T_{\text {max }}^{\mathcal{L}}\right\} \text { or } \arg \min _{\mathcal{L}}\left\{T_{a}^{\mathcal{L}}\right\}, & \text { otherwise. }\end{cases}$

\section{Coolest Path Routing}

Different from the routing problem in other wireless networks, the routing problem in cognitive radio networks is 


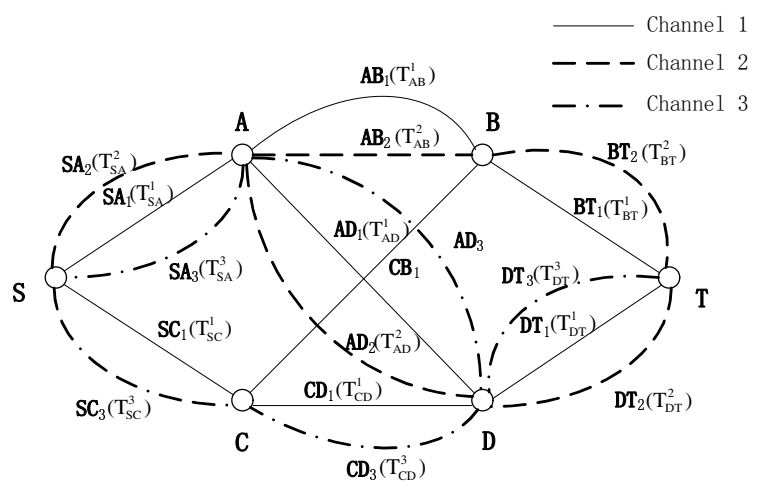

Figure 4. Topology of the cognitive radio network

composed of two subproblems, i.e., choosing an appropriate path and choosing the frequency band at each hop. The two subproblems are coupled with each other. So the routing algorithm in cognitive radio networks should address both subproblems. Using the path temperature as the routing metric explores spectrum diversity and potentially avoids the frequency bands and links with heavy traffic generated by the primary users. Stable path routing, or cool path routing achieves load balancing over both path and spectrum, because it prefers the paths and frequency bands with low spectrum utilization of primary users.

Routing in cognitive radio networks is actually composed of path selection and spectrum assignment. Therefore, the number of end-to-end paths increases with the number of channels in cognitive radio networks. Since each link is carried over multiple channels, the searching space of the optimal path is much larger than traditional networks. Fig. 4 shows the topology of a network with six cognitive radio nodes. Since each cognitive node has different communication capability, it can communicate over different set of channels. In this example, every node could communicate through two or three channels, shown in different line types. The number of common channels between each node pair varies from one to three. To show the possible paths in cognitive radio networks and facilitate solving the routing problem, we transform the topology $G=(V, E, C)$ into the routing graph $G_{R}=\left(V_{R}, E_{R}\right)$, as shown in Fig. 5, where $V$ and $V_{R}$ denote the vertex set of $G$ and $G_{R}$, respectively, $E$ and $E_{R}$ denote the edge set of $G$ and $G_{R}$, respectively, and $C$ is the common channel set of each edge in $E$. Since each link $e \in E$ is composed of multiple channels, we have $e=\left\{e_{c} \mid c \in c_{e}\right\}$, where $c_{e}$ is the common channel set over edge $e$. For example, the common channel set over edge $S A$ is $c_{S A}=\{1,2,3\}$. Therefore, link $S A=\left(S A_{1}, S A_{2}, S A_{3}\right)$. Each common channel between two nodes is defined as a sub-link. For example, $S A_{1}$ is a sub-link of $S A$. Each sublink is associated with its channel temperature specified in parentheses, such as the temperature of channel 2 between node $S$ and node $A$, say $T_{S A}^{2}$, in Fig. 4 .

For the convenience of analysis, we convert graph $G$ into

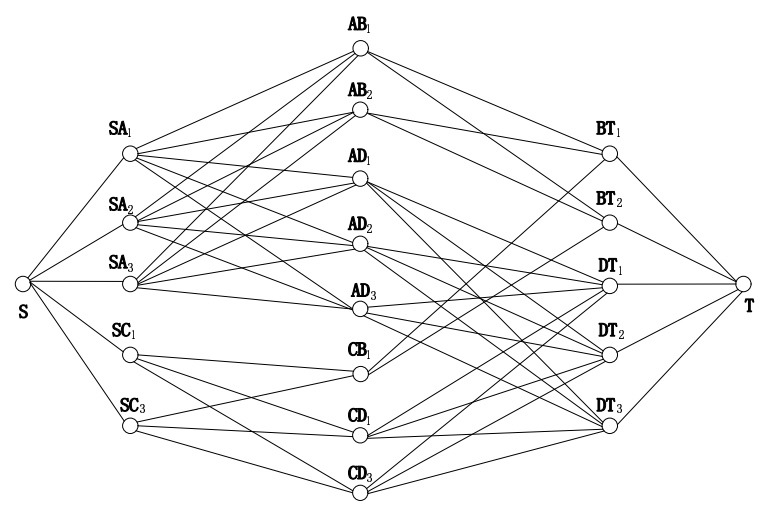

Figure 5. Routing graph of the cognitive radio network

routing graph $G_{R}$ by reducing the dimension of it. Each vertex in $G_{R}$ corresponds to a common channel over the edge $e_{c}$ in $G$. Consequently, the adjacency of the edges in $G$ is mapped into the adjacency of vertices in $G_{R}$. More specifically, for two adjacent edges in $E$, there is an edge joining the corresponding vertices in $V_{R}$. For example, adjacent edges $S A_{1}$ and $A B_{2}$ in $G$ are transformed into two adjacent vertices in $G_{R}$.

Apparently, the size of graph $G_{R}$ is greater than that of graph $G$. We have

$$
\left|V_{R}\right|=\sum_{e \in E}\left|c_{e}\right|=O\left(C_{\max }|E|\right),
$$

and

$$
\begin{aligned}
\left|E_{R}\right| & =\sum_{i \in V}\left(\sum_{k i \in E}\left(\left|c_{k i}\right| \cdot \sum_{i j \in E}\left|c_{i j}\right|\right)\right) \\
& \leq \sum_{i \in V}\left(\sum_{k i \in E}\left(C_{\max } \cdot \sum_{i j \in E} C_{\text {max }}\right)\right) \\
& =\sum_{i \in V} C_{\max }^{2}\left(\sum_{k i \in E} \sum_{i j \in E} 1\right) \\
& =O\left(C_{\text {max }}^{2}|E|^{2}|V|\right)
\end{aligned}
$$

where $C_{\max }$ is the maximum number of the channels supported at a node.

Eq. (7) shows the order of $G_{R}$, while Eq. (8) shows the size of $G_{R}$. The size of the routing graph is much greater than that of the topology, which grows with the number of channels. Consequently, the computational complexity of the routing algorithm in cognitive radio networks is much higher than in traditional networks.

Different routing metrics produce different optimal paths. First we propose routing algorithms based on the accumulated spectrum temperature metric and highest spectrum temperature metric, respectively. If the two algorithms yield the same optimal path, it means that there also exists an optimal path satisfying (6). 


\section{A. Minimum Accumulated Spectrum Temperature Path}

Given the routing graph, Dijkstra's algorithm can be applied to find the minimum accumulated spectrum temperature path. The computation complexity is $O\left(\left|V_{R}\right|^{2}\right)=$ $O\left(C_{\max }^{2}|E|^{2}\right)$. If there exist multiple optimal paths, one of them is randomly picked.

\section{B. Minimum Highest Spectrum Temperature Path}

To find the path with the minimum highest spectrum temperature, each node forwards the current highest spectrum temperature $T_{\max }$ to its neighboring node. When receiving the link information, current node compares the received $T_{\max }$ with the channel temperature $T_{i j}^{c}$, and update $T_{\max }$ as follows.

1) $T_{\max }$ does not change, if $T_{i j}^{c} \leq T_{\max }$. This means that there exists a sub-link with lower channel temperature than $T_{\text {max }}$.

2) $T_{\max }=T_{i j}$, if $T_{\max } \leq T_{i j}$. In this case, there exists no sub-link with lower channel temperature than $T_{\max }$, then $T_{\max }$ is updated with the link temperature.

We also apply Dijkstra's algorithm to find the minimum highest spectrum temperature path. So the computation complexity is $O\left(\left|V_{R}\right|^{2}\right)=O\left(C_{\max }^{2}|E|^{2}\right)$ as well. If multiple paths have the same minimum highest spectrum temperature, we randomly select one of them for routing.

\section{Minimum Mixed Spectrum Temperature Path}

Mixed path temperature is a two-dimensional metric, which consists of a bottleneck metric and an additive metric. If there exists an optimal path satisfying (6), then the minimum accumulated spectrum temperature path coincides with the minimum highest spectrum temperature path. So no more additional routing algorithm is necessary to find the path with the lowest path temperature. However, the routing problem is a multi-objective optimization problem and may not have a globally optimal solution. We construct a single aggregate objective function [10] to solve it. An ideal stable path has the minimum accumulated spectrum temperature with evenly distributed link temperature at each hop. Therefore, we solve the multi-objective optimization problem by using the following objective function

$$
\min _{\mathcal{L}} T_{m}^{\mathcal{L}}=T_{a}^{\mathcal{L}}+T_{\text {max }}^{\mathcal{L}}-\frac{T_{a}^{\mathcal{L}}}{H},
$$

where $H$ is the hop count. This objective function searches for the path with the minimum "temperature deviation" and accumulated spectrum temperature, which is defined as the mixed spectrum temperature.

To find the optimal path given the mixed temperature, $T_{\text {max }}^{\mathcal{L}}, T_{a}^{\mathcal{L}}$ and $h$ are propagated through the network. $T_{\text {max }}^{\mathcal{L}}$ and $T_{a}^{\mathcal{L}}$ are updated in the same way as in the previous algorithms. At the $h$ th hop, the mixed spectrum temperature $T_{m}^{\mathcal{L}, h}$ is updated as

$$
T_{m}^{\mathcal{L}, h}=\frac{h-1}{h}\left(T_{a}^{\mathcal{L}, h-1}+T^{\mathcal{L}, h}\right)+\max \left\{T_{m a x}^{\mathcal{L}, h-1}, T^{\mathcal{L}, h}\right\}
$$

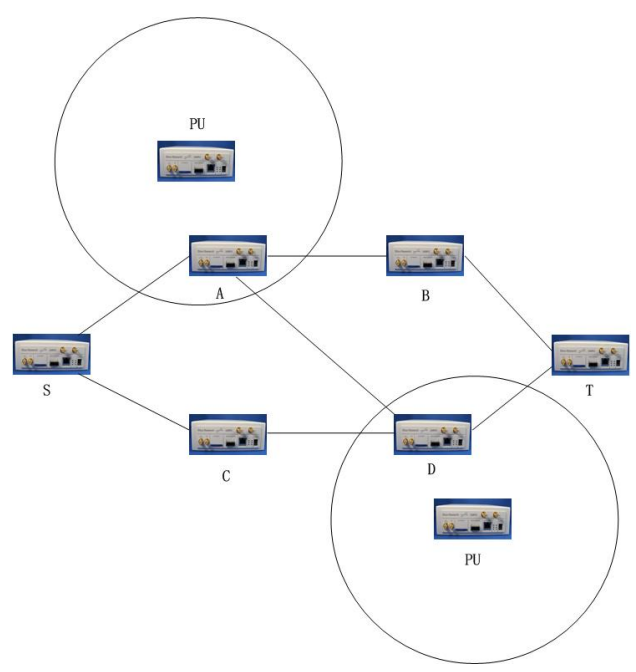

Figure 6. Network topology of testbed

where $T^{\mathcal{L}, h}$ is the link temperature at current node. Apparently, the mixed spectrum temperature $T_{m}^{\mathcal{L}, h}$ is nondecreasing. Dijkstra's algorithm is also applicable to find the path with the lowest mixed temperature.

\section{EXPERIMENTAL EVALUATION}

We investigate the performance of the proposed routing metrics in the software-defined radio based testbed, built with six USRP-2 devices and two primary nodes. We compare the performance of the three routing metrics, including accumulated spectrum temperature, highest spectrum temperature and mixed spectrum temperature, subject to the activities of the two primary users. We are interested in the relationship between the routing performance and the activities of the primary users. The effects of arrival rate of primary users and channel occupying time of primary users on the routing performance are investigated through extensive experiments. We introduce switch ratio and path longevity to show the path stability. The switch ratio is defined as the ratio of the number of route switches to the total number of PU appearances. The higher the switch ratio is, the worse the path stability is, and the more routing overhead would be. The path longevity is defined as the lifetime of a path, i.e., the time interval between establishing the path and ceasing the path usage. The longer the path longevity is, the better the path stability is achieved, and the less rerouting and path change would happen.

\section{A. Simulation Analysis}

In this part, we investigate the throughput of our proposed metrics compared with the random routing [11]. The random routing randomly forwards the packet towards the destination. The network topology is shown in Fig. 6. In our simulation, the value of the inter-arrival time of PU around node $D$ is set to 10 . As we can see from Fig. 7, the throughput of the four routing metrics increases while 


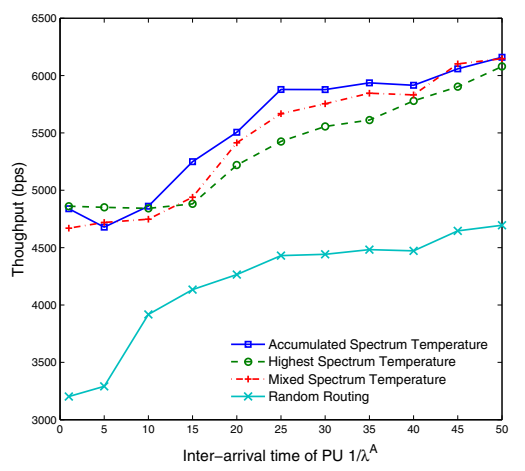

Figure 7. Throughput vs. the inter-arrival time of PU around node A

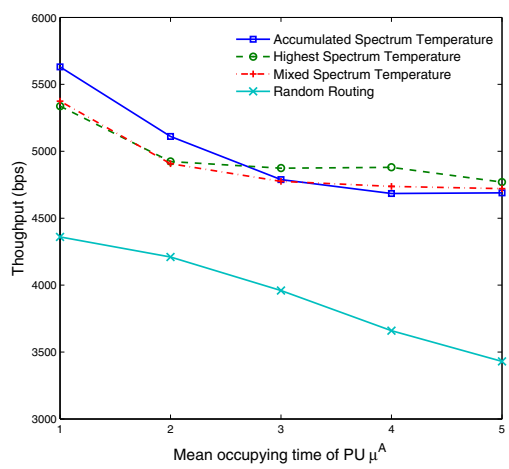

Figure 8. Throughput vs. the mean occupying time of PU around node $A$

the mean inter-arrival time of the PU around node $A$ rises. The number of route switches is reduced due to the longer interval of PU appearances, so the throughput is improved. Conversely, as shown in Fig. 8, the throughput decreases as the mean occupying time of PU around node $A$ rises. Obviously, the prolonged occupying time of PU leads to the depressed throughput of cognitive nodes. More importantly, the throughput of random routing is significantly lower than our proposed metrics. Our proposed metrics can improve the throughput by up to $51.7 \%$ in Fig. 7 and $36.6 \%$ in Fig. 8 than random routing. Without considering the PUs' activities, random routing is incapable of capturing the fluctuation of spectrum availability which induces dramatic drop in throughput. However, our proposed metrics are relatively immune to the variation of spectrum availability since they fully consider the impact of PUs' activities. In the next section, we will demonstrate our experimental result of the proposed metrics.

\section{B. Experiment Setup}

Each cognitive node transmits data packets over one of the three data channels, i.e. $2.43 \mathrm{GHz}, 2.45 \mathrm{GHz}$, and $2.48 \mathrm{GHz}$. We implement the routing metrics in AODV and use a common control channel to accommodate AODV. During the route discovery stage, each node updates the received path temperature information with its link temperature and broadcasts it to other nodes. When receiving all the path temperature information, the destination decides the optimal path and replies an ACK to the source in the reverse path.

In our experiments, we assume the arrival of the primary node follows a Poisson process. The channel occupying time of the primary user is exponentially distributed. When a primary node becomes active, the optimal path is recalculated. If the transmission is interrupted by a primary node, a new optimal path is selected. Otherwise, rerouting happens only when the optimal path changes. As the network topology shown in Fig. 6 , node $A$ and node $D$ are within the transmission range of the primary nodes. With different interference areas with respect to the PUs, the two nodes have different observations on the same channel. The source node $S$ generates packets destined to node $T$ at each second with a packet size of 2720 bits. The transmission rate of each sub-link is $500 \mathrm{kbps}$.

As shown in Fig. 6, all three paths are subject to the variation of spectrum availability. Path $S A B T$ and $S C D T$ are affected by the observations of node $A$ and node $D$ respectively. Path $S A D T$ is affected by the observations of both nodes. Therefore, the quality of the three paths is disparate.

\section{Effect of Inter-Arrival Time of Primary Nodes}

In the experiment, the appearance of the primary node on each channel, i.e. $2.43 \mathrm{GHz}, 2.45 \mathrm{GHz}$, and $2.48 \mathrm{GHz}$, is a Poisson process. Therefore, the inter-arrival times of PU are exponentially distributed with mean $1 / \lambda$ on each channel. The primary node occupies the channel for the time period which is exponentially distributed with parameter $1 / \mu=3$. To investigate the impact of the arrival rate of PU on routing, we let the mean inter-arrival time of PU around node $A$ over all channels, denoted $1 / \lambda^{A}$, varies from 1 to 50 with an interval 5 .

Fig. 9 (a), (b), (c) show the changes of the route switch ratio in accordance with the incremental mean inter-arrival time of the PU around node $A$ when the mean inter-arrival time of the PU neighboring node $D$, denoted as $1 / \lambda_{i}^{D}$, is fixed at 5, 10, 15, respectively. From Fig. 9 (a), we can see that, as the inter-arrival time of the PU increases, the switch ratio increases firstly, and then decreases. At first, the PU around $A$ appears frequently, so the source node prefers the path $S C D T$ not passing node $A$. As the inter-arrival time rises, the performance of the affected path $S A B T$ becomes preferable. Therefore, the path stability of $S A B T$ is boosted from poor to good, and then to excellence in comparison with the other paths. The same observation on the impact of the inter-arrival time can be obtained by comparing the three metrics in Fig. 9 (b) and (c).

The peak value of the switch ratio shows in which case the performance of the three metrics is highly comparable. As shown from Fig. 9 (a)-(c), the peak of the switch ratio 


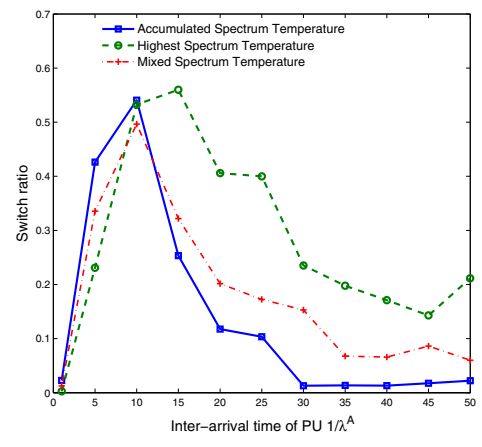

(a) $1 / \lambda_{i}^{D}=5$

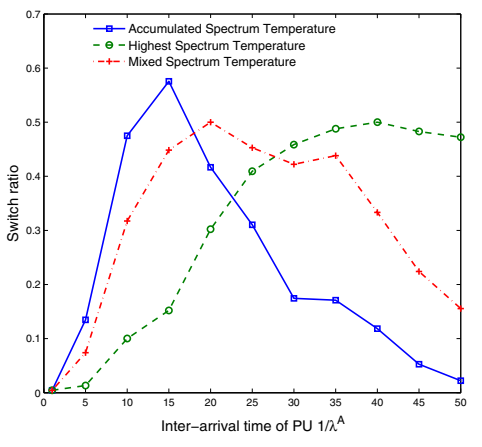

(b) $1 / \lambda_{i}^{D}=10$

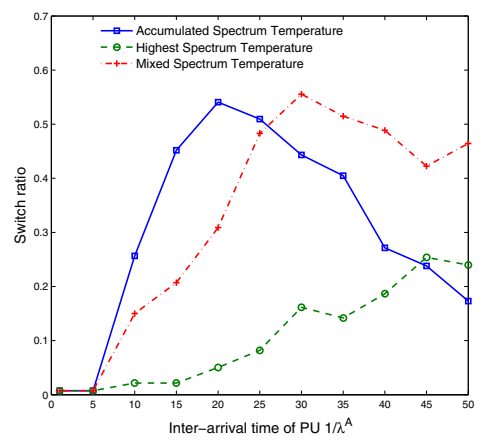

(c) $1 / \lambda_{i}^{D}=15$

Figure 9. Switch ratio vs. the inter-arrival time of the $\mathrm{PU}\left(1 / \lambda^{A}\right)$ around node $\mathrm{A}$.

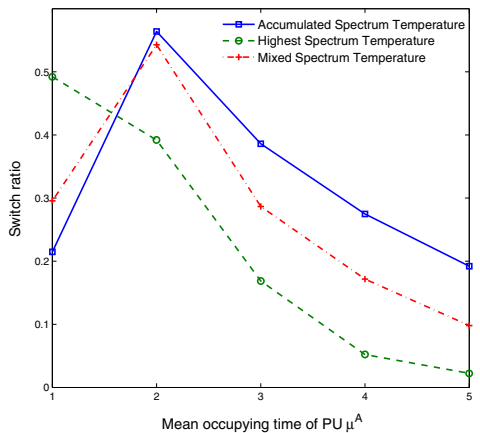

(a) $1 / \lambda_{i}^{A}=1 / \lambda_{i}^{D}=5$

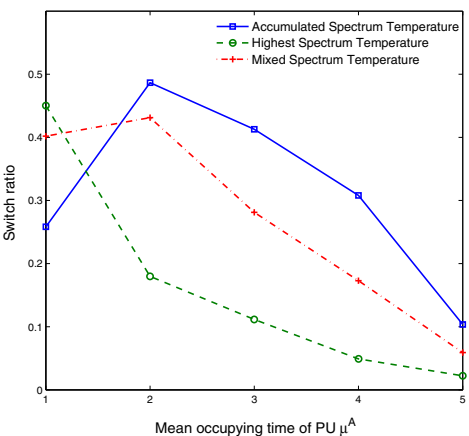

(b) $1 / \lambda_{i}^{A}=1 / \lambda_{i}^{D}=10$

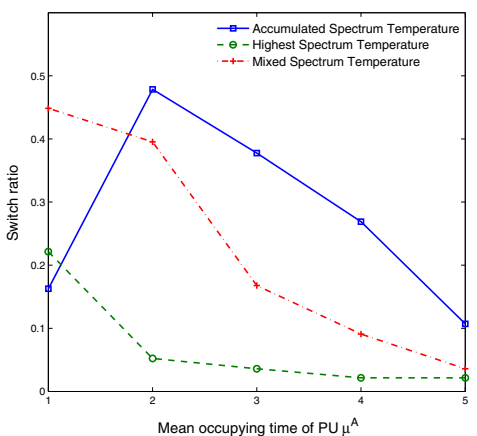

(c) $1 / \lambda_{i}^{A}=1 / \lambda_{i}^{D}=15$

Figure 10. Switch ratio vs. the mean occupying time of the $\mathrm{PU}\left(\mu^{A}\right)$ around node $A$.

moves towards greater value of mean inter-arrival time of PU as $1 / \lambda_{i}^{D}$ increases. This is because that when the mean inter-arrival time of the PU around node $A$ grows with the inter-arrival time of the PU around node $D$, the three paths achieve comparable path temperature. In addition, the overall performance of using the accumulated spectrum temperature is superior to others when the mean inter-arrival time around node $D$ equals 5 in Fig. 9 (a). The switch ratio of using the highest spectrum temperature is optimal when the inter-arrival time around node $D$ equals 15 in Fig. 9 (c). Therefore, in case of frequent PU appearances, the accumulated spectrum temperature may be more suitable than the other metrics. When the inter-arrival time increases, the highest spectrum temperature performs better.

\section{Effect of Channel Occupying Time of Primary Nodes}

The channel occupying time of the primary nodes is exponentially distributed with parameter $\mu$. The arrival rates of the primary users on the three channels follow a Poisson process with parameter $\lambda=5,10,15$, respectively. In this situation, the mean occupying time of the primary user around node $A$, denoted $\mu^{A}$, varies from 1 to 5 . We study the impact of the channel occupying time of PU on routing.

Fig. 10 (a), (b), (c) shows the route switch ratio in accordance with the incremental mean occupying time of the PU around node $A$ when the mean inter-arrival time of the PU around node $D$ is fixed at $5,10,15$, respectively. It is noted that the switch ratio in each figure decreases as the channel occupying time of PU increases. Obviously, the path across node $A$ worsens when the occupying time of PU increases. So the route switch ratio becomes lower since the source node only select the path with lower path temperature. The largest switch ratio indicates the point at which the path temperature of all paths is comparable, leading to frequent path switches. From Fig. 10 (a) - (c), we can see that the switch ratio corresponding to the highest spectrum temperature drops obviously with the increase of the inter-arrival time of the PU around node $D$. That means the highest spectrum temperature is more suitable for cognitive radio networks (CRNs) with longer inter-arrival time, or less PU appearances.

To summarize our experiments, the network performance greatly depends on the route switch ratio namely path stability. To enhance the network performance, we should select 
the path in a relatively stable state or relatively immune to spectrum variation. Each of our three metrics has pros and cons given different PU activities. Thus we should choose a metric with a lower switch ratio based on the arrival rate and the channel occupying time of PUs. For example, in Fig. 9 (a), when the PU arrival rate around node $D$ is $1 / 5$, we should choose accumulated spectrum temperature to find the path in a relatively stable state, thereby improving the path longevity and throughput. Also, according to Fig. 10, when the channel occupying time of PU increases, the highest spectrum temperature is preferable as it achieves better route switch ratio and path longevity.

In summary, The optimal path in CRNs should induces the lowest route switch ratio to maximize the throughput of cognitive nodes subject to PU activities. In case of frequent PU activities, the accumulated spectrum temperature achieves better performance in terms of path switch ratio, path longevity. When the PU arrival rate is low or channel occupying time of PU is long, the highest spectrum temperature performs better. The mixed spectrum temperature achieves compromised performance compared with the other two metrics.

\section{RELATED WORK}

Various routing metrics have been proposed in wireless networks, targeted at load-balancing [12]-[14] or characterizing channel interference [15]-[17]. Extensions of ETX [17] considering varying link reliability are introduced in [18]. Accounting for the impact of link positions, a new metric ETOP is developed to further improve ETX [19]. Incorporating achievable bandwidth using multiple radios into end-to-end delay, the weighted expected end-to-end delay metric better captures the effect of interference and channel diversity [7]. However, existing metrics are not readily applicable to cognitive radio networks because they do not consider the time-varying spectrum availability. Spectrum variation and spectrum mobility of SUs must be considered in designing routing metrics and algorithms. In this paper, we develop new routing metrics tailored to cognitive ad hoc networks which consider the spectrum variation at SUs.

Spectrum sharing and resource allocation have been studied to improve spectrum utilization, while routing is still an open area in cognitive radio networks. Shu and Krunz [20] address the truthful least-priced-path routing considering the supply-demand dynamics of the available spectrum. Some papers extend AODV or DSDV to support routing in cognitive networks. The authors in [21] extend AODV to find the path with the shortest delay, which includes channel switching delay and backoff delay. However, due to the long path discovery latency and heavy control overhead, it is not suitable for CRNs where PUs appear frequently. According to the instantaneous spectrum availability, the proposed routing protocol in [22] selects the path with the highest spectrum availability in a route mesh. The authors in [23] employ a centralized routing algorithm to find the path with the minimum delay or low channel switching probability based on the gathered global topology information. A route stability oriented protocol is presented in [24], where the maintenance of a route includes link switching and channel switching operations when PUs become active. Feng et. al [25] present an algorithm of joint handoff scheduling and routing in multi-hop CRNs. The routing algorithm tries to minimize the total link cost and ensure the connectivity along a path from source to destination. However, the routing overhead cannot be used as the sole indicator since CRNs are subject to various constraints, e.g., bottleneck and stability. In our proposed metrics, these factors are considered. Based on information-theoretic analysis, Chen et. al [26] propose approximate approaches to find the spectral efficient path in random networks. Experiment results in [27] show that cooperative relay could greatly improve the spectrum efficiency and network throughput by exploiting the diversity of spectrum resources. Joint routing and resource management is proposed to achieve optimal spectrum efficiency in the network with frequency-agile radios [28]. Adopting cooperative network coding in route selection, the throughput of unicast traffic can be improved in multi-rate wireless networks [29]. However, the works mentioned above are all based on the accurate spectrum sensing or prediction which is hard to implement in real world scenarios. Different from previous works, we propose routing metrics which can be relatively immune to the variation of spectrum utilization by choosing the statistically stablest path.

\section{CONCLUSION AND FUtURE WORK}

As spectrum handover caused by the appearance of primary users happens a lot in cognitive ad hoc networks, path breakage occurs frequently to the cognitive nodes. Existing routing metrics work for wireless networks with fixed operating channel sets. However, those routing metrics do not consider the spectrum dynamics, and hence are not suitable for cognitive radio networks. In this paper, we have proposed new routing metrics to capture the effects of the time-varying spectrum availability on routing in cognitive ad hoc networks. Experiment results on USRP-2 testbed demonstrate that using the proposed routing metrics could find the stablest path in cognitive ad hoc networks, thus achieving good network performance. Noticing that a common control channel may be not available in some scenarios, we will design routing algorithms without a common control channel in our future research.

\section{ACKNOWLEDGMENT}

This work was partially supported by the National Natural Science Foundation of China under grants No.60903192 and Guangdong S\&T Major Project under No.2009A080207002. The work of Fang was partially supported by the U.S. National Science Foundation under grants CNS-0916391 
and CNS-0721744, the National Natural Science Foundation of China under grant No. 61003300 and China 111 project under grant B08038 with Xidian University, Xi'an, China.

\section{REFERENCES}

[1] FCC, Spectrum policy task force report, FCC 02-155, Nov. 2002.

[2] J. Mitola, "Cognitive Radio: An Integrated Agent Architecture for Software Defined Radio," Ph. D Thesis, KTH Royal Institute of Technology, 2000.

[3] S. Haykin, "Cognitive Radio: Brain-Empowered Wireless Communications," IEEE Journal on Selected Areas in Communications, vol. 23, no. 2, pp. 201-220, Feb. 2005.

[4] I.F. Akyildiz, W. Lee, M. C. Vuran, and S. Mohanty, "A Survey on Spectrum Management in Cognitive Radio Networks", IEEE Communications Magazine, vol. 46, no. 4, pp. 40-48, Apr. 2008.

[5] A. P. Subramanian, H. Gupta, S. R. Das, and J. Cao, "Minimum Interference Channel Assignment in Multiradio Wireless Mesh Networks," IEEE Transactions on Mobile Computing, vol. 7, no. 12 , pp. $1459-1473$, Dec. 2008.

[6] B. Han, V. S. A. Kumar, M. V. Marathe, S. Parthasarathy, and A. Srinivasan, "Distributed Strategies for Channel Allocation and Scheduling in Software-Defined Radio Networks," Proc. of IEEE INFOCOM, pp. 1521-1529, 2009.

[7] H. Li, Y. Cheng, C. Zhou, and W. Zhuang, "Minimizing End-to-End Delay: A Novel Routing Metric for Multi-Radio Wireless Mesh Networks," Proc. of IEEE INFOCOM, pp. 4654, 2009.

[8] Y. Li, Y. Yang, and X. Lu, "Routing Metric Designs for Greedy, Face and Combined-Greedy-Face Routing," Proc. of IEEE INFOCOM, pp. 64-72, 2009.

[9] A. Anandkumar, N. Michael, and A. Tang, "Opportunistic Spectrum Access with Multiple Users: Learning under Competition," Proc. of IEEE INFOCOM, 2010.

[10] R. E. Steuer, Multiple Criteria Optimization: Theory, Computations, and Application, John Wiley \& Sons, 1986.

[11] I. F. Akyildiz, S. Weilian, Y. Sankarasubramaniam, and E. E. Cayirci, "A Survey on Sensor Networks," IEEE Communications Magazine, vol. 40, no. 8, pp. 102-114, 2002.

[12] A. Raniwala, T.-c. Chiueh, "Architecture and Algorithms for an IEEE 802.11-Based Multi-Channel Wireless Mesh Networks," Proc. of IEEE INFOCOM, pp. 2223-2234, 2005.

[13] J. So, N. H. Vaidya, "Load-Balancing Routing in Multichannel Hybrid Wireless Networks With Single Network Interface," IEEE Transactions on Vehicular Technology, vol. 56, no. 1, pp. 342-348, Jan. 2007.

[14] T. Liu and W. Liao, "Capacity-Aware Routing in MultiChannel Multi-Rate Wireless Mesh Networks," Proc. of ICC, pp. 1971-1976, 2006.
[15] H. Zhai and Y. Fang, "Impact of Routing Metrics on Path Capacity in Multi-rate and Multi-hop Wireless Ad Hoc Networks," Proc. of IEEE ICNP, pp. 86-95, 2007.

[16] D. S. J. D. Couto, D. Aguayo, J. Bicket, and R. Morris, "A High-Throughput Path Metric for Multi-Hop Wireless Routing," Proc. of ACM MobiCom, pp. 134-142, 2003.

[17] R. Draves, J. Padhye, and B. Zill, "Routing in Multi-Radio, Multi-Hop Wireless Mesh Networks," Proc. of ACM MobiCom, pp. 114-128, 2004.

[18] C. Koksal and H. Balakrishnan, "Quality-Aware Routing Metrics for Time-Varying Wireless Mesh Networks," IEEE Journal on Selected Areas in Communications, vol. 24, no. 11, pp. 1984-1994, Nov. 2006.

[19] G. Jakllari, S. Eidenbenz, N. Hengartner, S. V. Krishnamurthy, and M. Faloutsos, "Link Positions Matter: A Noncommutative Routing Metric for Wireless Mesh Networks," Proc. of IEEE INFOCOM, pp. 744-752, 2008.

[20] T. Shu and Marwan Krunz, "Truthful Least-Priced-Path Routing in Opportunistic Spectrum Access Networks," Proc. of INFOCOM, 2010.

[21] G. Cheng, W. Liu, Y. Li, and W. Cheng, "Spectrum Aware On-Demand Routing in Cognitive Radio Networks," Proc. of IEEE DySPAN, pp. 571-574, 2007.

[22] I. Pefkianakis, S. H. Y. Wong, and S. Lu, "SAMER: Spectrum Aware Mesh Routing in Cognitive Radio Networks," Proc. of IEEE DySPAN, 2008.

[23] S. Krishnamurthy, M. Thoppian, S. Venkatesan, and R. Prakash, "Control Channel Based MAC-Layer Configuration, Routing and Situation Awareness for Cognitive Radio Networks," Proc. of IEEE MILCOM, pp. 455-460, 2005.

[24] I. Filippini, E. Ekici, M. Cesana, "Minimum Maintenance Cost Routing in Cognitive Radio Networks," Proc. of IEEE MASS, pp. 284-293, 2009.

[25] W. Feng, J. Cao, C. Zhang, C. Liu, "Joint Optimization of Spectrum Handoff Scheduling and Routing in Multi-hop Multiradio Cognitive Networks," Proc. of IEEE ICDCS, pp. 85-92, 2009.

[26] D. Chen, M. Haenggi, and J. N. Laneman, "Distributed Spectrum-Efficient Routing Algorithms in Wireless Networks," IEEE Transactions on Wireless Communications, vol. 7, no. 2, pp. 5297-5305, Dec. 2008.

[27] J. Jia, J. Zhang, and Q. Zhang, "Cooperative Relay for Cognitive Radio Networks," Proc. of IEEE INFOCOM, pp. 2304-2312, 2009.

[28] Z. Feng and Y. Yang, "Joint Transport, Routing and Spectrum Sharing Optimization for Wireless Networks with FrequencyAgile Radios," Proc. of IEEE INFOCOM, pp. 1665-1673, 2009.

[29] J. Zhang and Q. Zhang, "Cooperative Network Coding-Aware Routing for Multi-Rate Wireless Networks," Proc. of IEEE INFOCOM 2009, pp. 181-189, 2009. 